\title{
On Stability in Impulsive Dynamical Systems
}

\author{
by
}

\author{
Krzysztof CIESIELSKI
}

Presented by Andrzej LASOTA

Summary. Several results on stability in impulsive dynamical systems are proved. The first main result gives equivalent conditions for stability of a compact set. In particular, a generalization of Ura's theorem to the case of impulsive systems is shown. The second main theorem says that under some additional assumptions every component of a stable set is stable. Also, several examples indicating possible complicated phenomena in impulsive systems are presented.

1. Preliminaries. Let $X$ be a metric space. A pair $(X, \pi)$ is a dynamical system if $\pi: \mathbb{R} \times X \rightarrow X$ is a continuous function with $\pi(0, x)=x$ and $\pi(t, \pi(s, x))=\pi(t+s, x)$ for every $t, s, x$; replacing $\mathbb{R}$ by $\mathbb{R}_{+}$we get the definition of a semidynamical system. For the elementary properties of dynamical and semidynamical systems, see $[\mathrm{BH}],[\mathrm{BS}],[\mathrm{NS}],[\mathrm{P}],[\mathrm{V}]$. We define the positive trajectory of $x$ as $\pi^{+}(x)=\pi([0,+\infty), x)=\pi([0,+\infty) \times\{x\})$.

In a semidynamical system, for $t \geq 0$ and $y \in X$ by $F(t, y)$ we mean $\{z \in X: \pi(t, z)=y\}$. In an analogous way we define $F(\Delta, D)$ for $\Delta \subset[0, \infty)$ and $D \subset X$. A point $x \in X$ is said to be a start point if $F(t, x)=\emptyset$ for all $t>0$.

An impulsive system $(X, \pi, M, I)$ consists of a semidynamical system $(X, \pi)$ (which may be a dynamical system), called the basic system, together with a nonempty closed subset $M$ of $X$ and a continuous function $I: M \rightarrow X$. We assume that for each $x \in M$ there is an $\varepsilon_{x}>0$ such that $\pi\left(\left(-\varepsilon_{x}, 0\right), x\right) \cap M=\emptyset$ and $\pi\left(\left(0, \varepsilon_{x}\right), x\right) \cap M=\emptyset$ (for dynamical systems), or $F\left(\left(0, \varepsilon_{x}\right), x\right) \cap M=\emptyset$ and $\pi\left(\left(0, \varepsilon_{x}\right), x\right) \cap M=\emptyset$ (for semidynamical systems). These conditions mean that the points of $M$ are isolated on every trajectory

2000 Mathematics Subject Classification: Primary 37B25; Secondary 54H20, 34A37.

Key words and phrases: dynamical system, flow, impulse, stability, invariance, prolongation. 
of the system $(X, \pi)$. We call $M$ the impulse set and $I$ the impulse function. By $M^{+}(x)$ we mean the set $\left(\pi^{+}(x) \cap M\right) \backslash\{x\}$.

We define a function $\phi: X \rightarrow(0,+\infty]$ by

$$
\phi(x)= \begin{cases}s & \text { if } \pi(s, x) \in M \text { and } \pi(t, x) \notin M \text { for } t \in(0, s), \\ +\infty & \text { if } M^{+}(x)=\emptyset\end{cases}
$$

(i.e. $\phi(x)$ is the smallest positive time for which the positive trajectory of $x$ meets $M)$. For $x \in M$ we call the point $\pi(\phi(x), x)$ the impulse point of $x$.

The trajectory $\widetilde{\pi}^{+}(x)$ of a point $x$ is defined as follows. We start from $x$. If $M^{+}(x)=\emptyset$ then we put $\tilde{\pi}(s, x)=\pi(s, x)$ for any $s \geq 0$. If $M^{+}(x) \neq \emptyset$ then we put $\widetilde{\pi}(s, x)=\pi(s, x)$ for $s<\phi(x)$ and $\tilde{\pi}(\phi(x), x)=I(\pi(\phi(x), x))$. Then we continue the above procedure starting at $\widetilde{\pi}(s, x)$ and so on. For $x \in X$, we denote by $x^{1}$ the point $\pi(\phi(x), x)$ and by $x^{1+}$ the point $\tilde{\pi}(\phi(x), x)=$ $I(\pi(\phi(x), x))=I\left(x^{1}\right)$. By $x^{2}$ we denote the point $\pi\left(\phi\left(x^{1+}\right), x^{1+}\right)$ and by $x^{2+}$ the point $\tilde{\pi}\left(\phi\left(x^{1+}\right), x^{1+}\right)=I\left(\pi\left(\phi\left(x^{1+}\right), x^{1+}\right)\right)=I\left(x^{2}\right)$ and so on. Thus, for any point $x \in X$ precisely one of the following three conditions holds:

(i) $M^{+}(x)=\emptyset$,

(ii) for some $n \geq 1: x^{k+}$ is defined for $k=1, \ldots, n$ and $M^{+}\left(x^{n+}\right)=\emptyset$,

(iii) for any $k \geq 1: x^{k+}$ is defined and $M^{+}\left(x^{k+}\right) \neq \emptyset$.

For any $x \in X$ we define the escape time $\widetilde{\omega}(x)$ of $x$ as $\sup \{s: \widetilde{\pi}(s, x)$ is defined\}.

Clearly, if $x$ satisfies (i) or (ii) then $\widetilde{\omega}(x)=+\infty$. If $x$ satisfies (iii) then either $\widetilde{\omega}(x)=+\infty$ or $\widetilde{\omega}(x) \in(0,+\infty)$. For a more detailed description, see [K1], [LBS], [C2].

By $\widetilde{\pi}^{+}(x)$ we mean the set $\widetilde{\pi}([0, \widetilde{\omega}(x)), x)$. By $\pi^{+}(A)$ we denote the set $\bigcup\left\{\pi^{+}(x): x \in A\right\}$; analogously we define $\widetilde{\pi}^{+}(A)$.

In a metric space $X$ with metric $d$ we write $B(x, \delta)$ for the ball with radius $\delta$ centred at $x$. By $B(A, \delta)$ we denote $\left\{x \in X: d_{A}(x)<\delta\right\}$, where $d_{A}(x)$ is the distance from $x$ to $A \subset X$. By $\mathcal{A}(A, \delta, \varepsilon)$ we denote the annulus $\{x \in$ $\left.X: \delta<d_{A}(x)<\varepsilon\right\}$. We say that a component $E$ of a closed set $A$ is isolated in $A$ if there exist disjoint open sets $U$ and $V$ such that $E \subset U$ and $A \backslash E \subset V$.

A point $x$ is stationary if $\pi(t, x)=x$ for all $t \geq 0$ (which is equivalent to $\widetilde{\pi}(t, x)=x$ for all $t \geq 0$ ). A point $x$ is periodic with respect to $\pi$ (or periodic in the system $(X, \pi))$ if $\pi(t, x)=x$ for some $t>0$ and $x$ is not stationary. A set $A \subset X$ is:

- positively $\pi$-invariant if $\pi^{+}(A) \subset A$;

- $\pi$-stable if for every $\varepsilon>0$ and $x \in A$ there exists a $\delta>0$ such that $\pi([0,+\infty), B(x, \delta)) \subset B(A, \varepsilon)$;

- $\pi$-orbitally stable if for every neighbourhood $U$ of $A$ there exists a positively $\pi$-invariant (not necessarily open) neighbourhood $V$ of $A$ with $V \subset U$; 
- $\pi$-stable in the sense of Bhatia and Hajek if for every $x \in A$ and $y \notin A$ there are a neighbourhood $V$ of $x$ and a neighbourhood $W$ of $y$ such that $W \cap \pi([0,+\infty), V)=\emptyset ;$

- a $\pi$-attractor if there exists an $\varepsilon>0$ such that $\lim _{t \rightarrow+\infty} d_{A}(\pi(t, x))=0$ for any $x \in B(A, \varepsilon)$.

The $\pi$-prolongation of $x \in X$ is the set $D^{+}(x)=\{y \in X$ : there are sequenes $\left(x_{n}\right),\left(t_{n}\right)$ such that $x_{n} \rightarrow x, t_{n} \geq 0$ for each $n$ and $\left.\pi\left(t_{n}, x_{n}\right) \rightarrow y\right\}$. We put $D^{+}(B)=\bigcup\left\{D^{+}(x): x \in B\right\}$. In an analogous way we define the corresponding notions for $\tilde{\pi}$.

We say that an impulsive system satisfies (STC) (Strong Tube Condition) if for any point $x \in M$ there exists a section $S$ through $x$ with a tube $U$ such that $S=M \cap U$ (cf. [BS], [NS], [C1], [C2], [K1]).

We say that $A$ is $I$-invariant in the impulsive system if $I(x) \in A$ for each $x \in M \cap A$; and $A$ is $I$-stable if for every $\varepsilon>0$ there exists a $\delta>0$ such that $I(M \cap B(A, \delta)) \subset B(A, \varepsilon)$.

Throughout this paper we assume that $(X, \pi, M, I)$ (written as $(X, \tilde{\pi})$ ) is the impulsive system given by a semidynamical system $(X, \pi)$ (which may be a dynamical system) on a metric space $X$.

2. Invariance. We start from some remarks on invariance. It can be easily checked that none of three types of invariance: $\pi$-invariance, $\widetilde{\pi}$-invariance and $I$-invariance implies any other one. It is enough to consider a system on $\mathbb{R}$ such that $\pi(t, x)=t+x, M=\{1\}, I(1)=-1$. Taking the sets $[0,+\infty),[-1,1),[-1,1],[-2,2]$ and $[1,+\infty)$ we get all the required examples. However, we have

2.1. Proposition. Any positively $\pi$-invariant and $I$-invariant set $A$ is positively $\tilde{\pi}$-invariant.

Proof. Let $x \in A$. Then $\tilde{\pi}([0, \phi(x)), x)=\pi([0, \phi(x)), x) \subset A$ and $x^{1}=$ $\pi(\phi(x), x) \in A$. Therefore $x^{1+}=I\left(x^{1}\right) \in A$ as $A$ is $I$-invariant. In the same manner we get $\tilde{\pi}\left(\left[\phi(x), \phi(x)+\phi\left(x^{1}\right)\right), x\right)=\pi\left(\left[0, \phi\left(x^{1}\right)\right), x^{1}\right) \subset A$ and $x^{2} \in A$. We continue in this fashion obtaining $\tilde{\pi}^{+}(x) \subset A$.

2.2. Proposition. Any positively $\tilde{\pi}$-invariant closed set is positively $\pi$ invariant.

Proof. Let $x \in A$. Suppose that $\pi(s, x) \notin A$ for some $A$ and define $t=$ $\inf \{s: \pi(s, x) \notin A\}$. Then $t>0$ as $\tilde{\pi}([0, \phi(x)), x)=\pi([0, \phi(x)), x) \subset A$. We have $\pi([0, t), x) \subset A$ and $\pi(t, x) \in A=\bar{A}$. However, $\pi([0, \phi(\pi(t, x))), \pi(t, x))$ $=\tilde{\pi}([0, \phi(\pi(t, x))), \pi(t, x)) \subset A$, so $\pi([0, t+\delta), x) \subset A$ for some $\delta>0$, which contradicts the definition of $t$.

Any $\pi$-stable compact set is positively $\pi$-invariant (cf. [BH], [BS], $[\mathrm{NS}],[\mathrm{P}])$. For $\tilde{\pi}$-stability we have 
2.3. TheOrem. Any compact $\widetilde{\pi}$-stable set $A$ is $\widetilde{\pi}$-invariant and $\pi$-invariant. If there is no start point in $M \cap A$, then $A$ is also I-invariant.

Proof. Take an $\varepsilon>0$. For any $x \in A$ there is a $\delta>0$ such that $\tilde{\pi}^{+}(B(x, \delta)) \subset B(A, \varepsilon)$, so $\tilde{\pi}^{+}(x) \subset \bigcap\{B(A, \varepsilon): \varepsilon>0\}=\bar{A}=A$.

The second property follows from the previous one and Proposition 2.2.

Now let $x \in M \cap A$; there are $y \in X, t>0$ and $u>t$ such that $\pi(t, y)=x$ and $\pi([0, u], y) \cap M=\{x\}$. Take any $\varepsilon>0$. There exists a $\delta>0$ such that $\tilde{\pi}^{+}(B(x, \delta)) \subset B(A, \varepsilon)$. We can find an $s \in[0, t)$ such that $\pi(s, y) \in B(x, \delta)$. Thus $I(x)=I(\pi(t-s, \pi(s, y)))=\widetilde{\pi}(t-s, \pi(s, y)) \in B(A, \varepsilon)$. Therefore $I(x) \in \bigcap\{B(A, \varepsilon): \varepsilon>0\}=\bar{A}=A$.

2.4. REMARK. In particular, the condition on start points is always fulfilled in the case of dynamical systems. Even for semidynamical systems, the situation where there is a start point in $M \cap A$ is rather strange; however, it may happen. In that case $I$ is formally defined for such a point but in fact has practically nothing in common with the impulsive system as the movement of this point in the system $(X, \widetilde{\pi})$ is given by $\pi$; moreover, there is no way to get to this point.

2.5. TheOrem. Assume that a component $E$ of a compact positively $\widetilde{\pi}$-invariant set $A$ is $I$-invariant. Then $E$ is positively $\widetilde{\pi}$-invariant.

Proof. Let $x \in E$. Then $\pi([0, \phi(x)), x) \subset A$, as according to Theorem 2.3, $A$ is positively $\pi$-invariant. Therefore $\pi([0, \phi(x)), x) \subset E$. If $\phi(x)=+\infty$, the proof is finished. If $\phi(x)<+\infty$, then $x^{1}=\pi(\phi(x), x) \in \bar{E}=E$ and $I\left(x^{1}\right) \in E$. We proceed in this manner to get $\widetilde{\pi}^{+}(x) \subset E$.

3. Stability in impulsive systems and basic systems. We start from some examples which show that the connection between stability in a basic system and in an impulsive system can be very week.

3.1. ExAmple. We show an example of a $\widetilde{\pi}$-stable and $\pi$-invariant set which is not $\pi$-stable. Take $X=\{(r, \theta): r \geq 1\} \subset \mathbb{R}^{2}$ with polar coordinates. Consider the system given by the equations $r^{\prime}=(r-1) / 100, \theta^{\prime}=1$ with

$$
M=[1,+\infty) \times\left\{\frac{\pi}{2}\right\}, \quad I(r, 0)=\left(\frac{r+99}{100}, \frac{3 \pi}{2}\right)
$$

(see Figure 1). The unit circle $A=\{(r, \theta): r=1\}$ is not $\pi$-stable as the trajectories of the basic system go spirally from the unit circle to infinity. However, $A$ is $\widetilde{\pi}$-stable because in the impulsive system a moving point "jumps down" from the upper vertical half-line onto the lower vertical halfline; after an impulse it is closer to the unit circle; when it comes again to the upper half-line it is closer to the circle than at the moment of the previous jump; then it jumps again. 


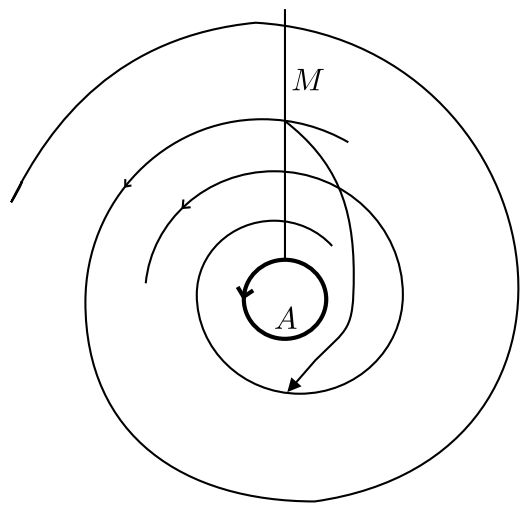

Fig. 1

3.2. Example. We exhibit a set $A$ which is not $\widetilde{\pi}$-stable, although it is $\pi$-stable, $\widetilde{\pi}$-positively invariant, $I$-invariant, $I$-stable and it is even a $\widetilde{\pi}$-attractor (in particular, there is an $\varepsilon>0$ such that $d_{A}\left(x^{n}\right) \rightarrow 0$ and $d_{A}\left(x^{n+}\right) \rightarrow 0$ for any $x \in B(x, \varepsilon)$ with infinite number of impulses).

Take $X=\{(r, \theta): 1 \leq r \leq 2\} \subset \mathbb{R}^{2}$ with polar coordinates. Consider the system given by the equations $r^{\prime}=0, \theta^{\prime}=1$ and take $M_{1}=[1,3 / 2] \times\{\pi / 2\}$, $M_{2}=[1,2] \times\{3 \pi / 2\}$ and $M=M_{1} \cup M_{2}$. For $x \in M_{1}$ we put $I(r, \pi / 2)=$ $(2 r-1,0)$ and for $x \in M_{2}$ we put $I(r, 3 \pi / 2)=((r+1) / 2, \pi)$ (see Figure 2$)$.

The unit circle $A=\{(r, \theta): r=1\}$ is $\pi$-stable and $I$-stable. Each point of $X$ is attracted to $A$, because after some time it falls into $M_{2}$ and then it will approach $A$. However, $A$ is not $\widetilde{\pi}$-stable since all the points of $\{(r, \theta): 1<r<3 / 2,0<\theta<\pi / 2\}$ fall after some time into the annulus $\{(r, \theta): 3 / 2 \leq r \leq 2\}$.

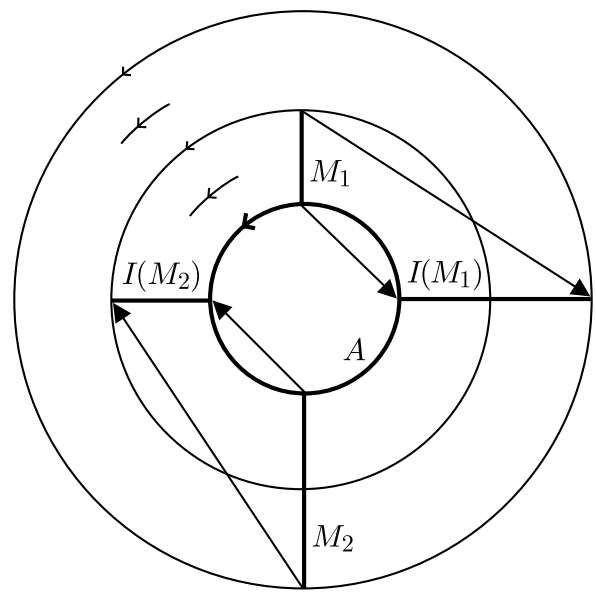

Fig. 2 
To get a system fulfilling (STC) it is enough to take $M=M_{1} \cup M_{2} \cup M_{3}$, where $M_{3}=[3 / 2,2] \times\{\pi / 2\}$ and to map $M_{3}$ onto $I\left(M_{3}\right)=\{2\} \times[0, \pi]$ with $I(3 / 2, \pi / 2)=(2,0)$.

However, some results can be proved. We have

3.3. Theorem. Assume that $X$ is locally compact and $A$ is a compact $\pi$-stable subset of $X$. Assume also that there is a $\varrho$ such that for every $x \in B(A, \varrho)$ the following conditions hold:

(a) if $x \in M$ then $d_{A}(I(x)) \leq d_{A}(x)$,

(b) if $x \in I(M)$ and $\phi(x)<+\infty$, then $d_{A}(\pi(\phi(x), x)) \leq d_{A}(x)$.

Then $A$ is $\widetilde{\pi}$-stable.

Proof. Take an $\varepsilon>0$; we may assume that $\varepsilon<\varrho$. To prove the $\widetilde{\pi}$-stability of $A$ it is enough to show that there is a $\delta>0$ such that $\widetilde{\pi}^{+}(x) \subset B(A, \varepsilon)$ for any $x \in B(A, \delta)$.

From the $\pi$-stability of $A$ we can find an $\eta>0$ such that $\pi(B(A, \eta)) \subset$ $B(A, \varepsilon)$, since $A$ is compact. Similarly, there is a $\delta>0$ such that $\pi(B(A, \delta))$ $\subset B(A, \eta / 2)$. Now take an $x \in B(A, \delta)$. If there is no impulse point in $\widetilde{\pi}^{+}(x)$ we have $\widetilde{\pi}^{+}(x)=\pi^{+}(x) \subset B(A, \varepsilon)$. Assume then that $\phi(x)<+\infty$. We have $\tilde{\pi}([0, \phi(x)), x)=\pi([0, \phi(x)), x) \subset B(A, \eta / 2) \subset B(A, \varepsilon) \subset B(A, \varrho)$ so $x^{1}=\pi(\phi(x), x) \in \overline{B(A, \eta / 2)}$ and $d_{A}\left(x^{1}\right) \leq \eta / 2<\eta<\varrho$. Thus $d_{A}\left(x^{1+}\right) \leq$ $\eta / 2$ and $x^{1+} \in B(A, \eta)$, so $\tilde{\pi}\left(\left[\phi(x), \phi\left(x^{1+}\right)\right), x\right)=\pi\left(\left[0, \phi\left(x^{1+}\right)\right), x^{1+}\right) \subset$ $B(A, \varepsilon)$ and $d_{A}\left(\pi\left(\phi\left(x^{1+}\right), x^{1+}\right)\right) \leq d_{A}\left(x^{1+}\right) \leq \eta / 2$. Therefore $d_{A}\left(x^{2+}\right) \leq$ $d_{A}\left(x^{2}\right) \leq \eta / 2$. We continue in this fashion to obtain $d_{A}\left(x^{n}\right) \leq \eta / 2, d_{A}\left(x^{n+}\right)$ $\leq \eta / 2, \pi\left(\left[0, \phi\left(x^{n+}\right)\right), x^{n+}\right) \subset B(A, \varepsilon)$. We conclude that $\widetilde{\pi}^{+}(x) \in B(A, \varepsilon)$.

We also have a simple

3.4. Proposition. Assume that $A$ is a compact $\pi$-stable subset of $X$ and $A \cap M=\emptyset$. Then $A$ is $\widetilde{\pi}$-stable.

Proof. There is a $\beta>0$ such that $B(A, \beta) \cap M=\emptyset$ as $A$ is compact and $M$ is closed. Take any $\varepsilon>0$. For any $x \in A$ there is a $\delta^{\prime}>0$ with $\pi^{+}\left(B\left(x, \delta^{\prime}\right)\right) \subset B(A, \varepsilon)$. If we take $\delta=\min \left\{\delta^{\prime}, \beta\right)$ we have $\widetilde{\pi}^{+}\left(B\left(x, \delta^{\prime}\right)\right) \subset$ $B(A, \varepsilon)$.

The stability of stationary and periodic trajectories is of particular interest. We have the immediate

3.5. Corollary. If $x \notin M$ is a stationary point and $\{x\}$ is a $\pi$-stable set then $\{x\}$ is a $\widetilde{\pi}$-stable set.

3.6. Corollary. If $x$ is periodic with respect to $\widetilde{\pi}, \pi^{+}(x) \cap M=\emptyset$ and $\pi^{+}(x)$ is a $\pi$-stable set, then $\pi^{+}(x)=\widetilde{\pi}^{+}(x)$ is a $\tilde{\pi}$-stable set.

We also have 
3.7. ThEOREM. Assume that a compact set $A$ is $\pi$-stable and there exists $a \varrho>0$ such that $I(M \cap B(A, \varrho)) \subset A$. Then $A$ is $\tilde{\pi}$-stable.

Proof. Take an $\varepsilon>0$; we may assume that $\varepsilon<\varrho$. From the compactness and stability of $A$ there is a $\delta>0$ such that $\pi^{+}(B(A, \delta)) \subset B(A, \varepsilon)$. We show that $\tilde{\pi}^{+}(B(A, \delta)) \subset B(A, \varepsilon)$. Take an $x \in B(A, \delta)$. If there is no impulse point in $\tilde{\pi}^{+}(x)$ we have $\tilde{\pi}^{+}(x)=\pi^{+}(x) \subset B(A, \varepsilon)$.

Assume now that $\phi(x)<+\infty$. Then $\tilde{\pi}([0, \phi(x)), x) \subset B(A, \varepsilon), x^{1}=$ $\pi(\phi(x), x) \in B(A, \varrho)$ and $x^{1+} \in A \subset B(A, \delta)$. We continue in this fashion obtaining $\tilde{\pi}\left(\left[0, \phi\left(x^{1+}\right)\right), x^{1+}\right) \subset B(A, \varepsilon)$ and $x^{2+} \in A \subset B(A, \delta)$. We proceed analogously to get $\tilde{\pi}^{+}(x) \subset B(A, \varepsilon)$.

4. Conditions for stability of compact sets. For dynamical systems, there are some criteria for stability of compact sets. Impulsive systems may have more complicated motion, even for simple systems. Surprisingly enough, some results analogous to those for dynamical systems hold. In particular, Ura's famous theorem connecting the stability of a compact set with a property of the prolongation is true. The proof is not a simple analogy of that for dynamical systems. This is, in particular, the case for the implication $(4) \Rightarrow(1)$ of the following theorem.

4.1. TheOrem. Assume that $X$ is locally compact and $A$ is a compact subset of $X$. Then the following conditions are equivalent:

(1) $A$ is $\tilde{\pi}$-stable.

(2) $A$ is $\tilde{\pi}$-orbitally stable.

(3) $A$ is $\tilde{\pi}$-stable in the sense of Bhatia and Hajek.

(4) $\widetilde{D}^{+}(A)=A$.

(The equivalence $(1) \Leftrightarrow(4)$ is Ura's Theorem for impulsive systems.)

Proof. $(1) \Rightarrow(2)$. Take a neighbourhood $U$ of $A$. There is a neighbourhood $V$ of $A$ with $V \subset \widetilde{\pi}^{+}(V) \subset U$. Then $\widetilde{\pi}^{+}(V)$ is the required positively invariant neighbourhood of $A$.

$(2) \Rightarrow(3)$. Take $x \in A$ and $y \notin A$. We can find open sets $W$ and $V$ with $W \supset A, y \in V, W \cap V=\emptyset$. From (2) we can find a positively invariant neighbourhood $U$ of $A$ such that $U \subset W$. The sets Int $U$ and $V$ fulfill the conditions of the definition of stability in the sense of Bhatia and Hajek.

$(3) \Rightarrow(4)$. Clearly, $A \subset \widetilde{D}^{+}(A)$. We show that $\widetilde{D}^{+}(A) \subset A$. Take $y \notin A$ and $x \in A$. There are neighbourhoods $U$ of $x$ and $V$ of $y$ with $\tilde{\pi}^{+}(U) \cap V=\emptyset$. Then for any sequences $\left(x_{n}\right)$ and $\left(t_{n}\right)$ such that $x_{n} \rightarrow x$ and $t_{n} \geq 0$ we have $x_{n} \in U, \tilde{\pi}\left(t_{n}, x_{n}\right) \in U, \tilde{\pi}\left(t_{n}, x_{n}\right) \notin V$ for $n \geq n_{0}$ and thus $\tilde{\pi}\left(t_{n}, x_{n}\right) \nrightarrow y$. This shows that for any $x \in A$ we have $y \notin \widetilde{D}^{+}(x)$ and finally $y \notin \widetilde{D}^{+}(A)$. 
$(4) \Rightarrow(1)$. Suppose to the contrary that there are $\varepsilon>0$ and sequences $\left(x_{n}\right),\left(t_{n}\right)$ such that $x_{n} \rightarrow x, t_{n} \geq 0$ and $\tilde{\pi}\left(t_{n}, x_{n}\right) \notin \overline{B(A, \varepsilon)}$. We may assume that $\overline{B(A, \varepsilon)}$ is compact as $X$ is locally compact.

Assume that $t_{n}<\widetilde{\omega}\left(x_{n}\right)$ for infinitely many $n$. Taking a subsequence we may assume that $t_{n}<\widetilde{\omega}\left(x_{n}\right)$ for every $n$. Then $\widetilde{\pi}\left(t_{n}, x_{n}\right)=\pi\left(t_{n}, x_{n}\right)$ and for each $n$ there is an $s_{n} \in\left[0, t_{n}\right]$ with $\widetilde{\pi}\left(s_{n}, x_{n}\right)=\pi\left(s_{n}, x_{n}\right) \in \partial B(A, \varepsilon)$. The last set is compact, so $\widetilde{\pi}\left(s_{n_{k}}, x_{n_{k}}\right) \rightarrow y \in \partial B(A, \varepsilon)$ for some subsequence $\left(n_{k}\right)$. Thus $y \in \widetilde{D}^{+}(A) \backslash A$, which is a contradiction. We now need to prove the theorem in the case where $t_{n}<\widetilde{\omega}\left(x_{n}\right)$ only for finitely many $n$.

We may assume that $\widetilde{\omega}\left(x_{n}\right) \leq t_{n}$ for all $n$ (in other words, each $\widetilde{\pi}\left(t_{n}, x_{n}\right)$ appears on the trajectory $\widetilde{\pi}^{+}\left(x_{n}\right)$ later than the impulse point of $\left.x_{n}\right)$.

Set $\Delta_{n}=\left\{s \geq 0: \widetilde{\pi}\left(s, x_{n}\right) \in B(A, \varepsilon)\right\}$. Note that $0 \in \Delta_{n}$. Thus we may define $s_{n}=\inf \left[0, \widetilde{\omega}\left(x_{n}\right)\right) \backslash \Delta_{n}$. Notice that $\widetilde{\pi}\left(s_{n}, x_{n}\right) \notin B(A, \varepsilon)$ as the function $\widetilde{\pi}\left(\cdot, x_{n}\right)$ is right-continuous.

Now take a $\delta \in(0, \varepsilon)$. We show that there is an $n_{0}$ such that for any $n \geq n_{0}$ we can find $p_{n} \in \widetilde{\pi}\left(\Delta_{n}, x_{n}\right)$ and $q_{n} \in A$ such that $d\left(p_{n}, q_{n}\right)<$ $\delta$. Suppose not. Then for infinitely many $n$ we can find a $u_{n} \in \Delta_{n}$ with $\tilde{\pi}\left(u_{n}, x_{n}\right) \in \overline{\mathcal{A}(A, \delta, \varepsilon)}$. The last set is compact, so for some subsequence $\left(u_{n_{k}}\right)$ of $\left(u_{n}\right)$ we have $\widetilde{\pi}\left(u_{n_{k}}, x_{n_{k}}\right) \rightarrow y \notin A$, which is impossible as $\widetilde{D}^{+}(A)=A$.

For any fixed $n \geq n_{0}$ we have $\widetilde{\pi}\left(\left[0, s_{n}\right), x_{n}\right) \subset B(A, \delta)$ and $\widetilde{\pi}\left(s_{n}, x_{n}\right) \notin$ $B(A, \varepsilon)$, which means that $\widetilde{\pi}\left(\cdot, x_{n}\right)$ is not continuous at $s_{n}$, so $\widetilde{\pi}\left(s_{n}, x_{n}\right)=$ $I\left(y_{n}\right)$ for some $y_{n} \in M$. Moreover, $y_{n} \in \overline{B(A, \delta)}$, because $\widetilde{\pi}\left(s, x_{n}\right) \rightarrow y$ as $s \nearrow s_{n}$. Note also that $y_{n}$ is not a start point as $s_{n}>0$.

Using the compactness of $\overline{B(A, \delta)}$ we may assume that $y_{n} \rightarrow y \in \overline{B(A, \delta)}$. From the continuity of $I$ we get $I\left(y_{n}\right) \rightarrow I(y)$, which gives $I(y) \notin A$, because $I\left(y_{n}\right) \notin B(A, \varepsilon)$ for every $n$. On the other hand, $I\left(y_{n}\right)=\widetilde{\pi}\left(s_{n}, x_{n}\right)$, which means that $I\left(y_{n}\right)=\lim _{n \rightarrow \infty} \widetilde{\pi}\left(s_{n}, x_{n}\right) \in \widetilde{D}^{+}(x) \subset \widetilde{D}^{+}(A)$. This contradicts the assumption $\widetilde{D}^{+}(A)=A$ and finishes the proof.

5. Stability of components. For a dynamical system, a compact set $A$ is stable if and only if each component of $A$ is stable. This does not hold for impulsive systems.

5.1. EXAMPLE. Consider the planar dynamical system given by the equations $x^{\prime}=-y, y^{\prime}=x$. Here each point is periodic except $(0,0)$ which is a stationary point. Let $A_{1}=\left\{(x, y): x^{2}+y^{2}=1\right\}, A_{2}=\left\{(x, y): x^{2}+y^{2}=4\right\}$, $A=A_{1} \cup A_{2}$. Let $M=\{(0,-2),(0,-1)\}, I((0,-2))=(0,1), I((0,-1))$ $=(0,2)$. It is easy to see that $A$ is stable, but no component of $A$ (i.e. neither $A_{1}$ nor $A_{2}$ ) is stable. 
5.2. ExAMPLE. It is easy to modify the above example to get a system satisfying (STC). Consider the restriction of the basic system from the previous example to the set $X=\left\{(x, y): 1 \leq x^{2}+y^{2} \leq 4\right\}$ and take $M=\{0\} \times[-2,-1]$ with $I((0, y))=(0, y+3)$. Here again $A$ is stable, but neither $A_{1}$ nor $A_{2}$ is. Note that in this impulsive system for $x \leq 0$ any point $(x, y)$ is periodic, while for $x>0$ a point $(x, y)$ is eventually periodic. This example shows that the condition (STC), though important in the theory of impulsive systems for several reasons, has nothing to do with stability of components.

However, we have an easy

5.3. Proposition. Assume that $A$ is compact and any component of $A$ is $\widetilde{\pi}$-stable. Then $A$ is $\widetilde{\pi}$-stable.

Proof. Let $U$ be a neighbourhood of $A$. Take any $x \in A$. Let $A_{x}$ be a component of $A$ such that $x \in A_{x}$. Of course $U$ is a neighbourhood of $A_{x}$. Thus there exists a neighbourhood $V_{x}$ of $x$ such that $\tilde{\pi}^{+}\left(V_{x}\right) \subset U$. This finishes the proof.

Examples 5.1 and 5.2 suggest that the reason why a $\widetilde{\pi}$-stable set need not have stable components is that the component need not be $I$-invariant. Indeed, we have

5.4. TheOrem. Let $X$ be locally compact. Assume that an isolated component $E$ of a compact $\widetilde{\pi}$-stable set $A$ is $I$-invariant. Then $E$ is $\widetilde{\pi}$-stable.

Proof. We may assume that $\bar{U}$ is compact. Suppose to the contrary that there exist a neighbourhood $W$ of $B$, an $x \in E$ and a sequence $\left(x_{n}\right)$ such that $x_{n} \rightarrow x$ and $\tilde{\pi}^{+}\left(x_{n}\right)$ is not contained in $W$. We may assume that $W \subset U$ (thus, $W$ is compact).

There exists an $\varepsilon>0$ such that $\overline{B(E, \varepsilon)} \subset W$ as $E$ is compact. Hence $\overline{B(E, \varepsilon)}$ is compact.

First notice that $M^{+}\left(x_{n}\right) \neq \emptyset$ for each such $n$. If not, for any $n$ there is a $u_{n} \geq 0$ such that $\tilde{\pi}\left(u_{n}, x_{n}\right)=\pi\left(u_{n}, x_{n}\right) \in \partial B(E, \varepsilon)$. Taking a subsequence we have $\tilde{\pi}\left(u_{n}, x_{n}\right) \rightarrow y \in \partial B(E, \varepsilon)$; so $y \in \widetilde{D}^{+}(x) \subset \widetilde{D}^{+}(A)$ and $y \notin A$. This is impossible according to Theorem 4.1.

There is a neighbourhood $G_{x}$ of $x$ such that $\tilde{\pi}^{+}\left(G_{x}\right) \subset B(E, \varepsilon) \cup V$, because $B(E, \varepsilon) \cup V$ is a neighbourhood of the stable set $A$. For $n$ large enough we have $x_{n} \in G_{x}$. Using the property shown above we know that for each $n$ the set $\left\{t: \tilde{\pi}\left(t, x_{n}\right) \in V\right\}$ is nonempty. Define $t_{n}=\inf \left\{t: \tilde{\pi}\left(t, x_{n}\right) \in V\right\}$. Thus $\tilde{\pi}\left(\left[0, t_{n}\right), x_{n}\right) \subset B(E, \varepsilon)$. According to the construction of impulsive trajectories and the property that the points of $M$ are isolated on every trajectory of the system $(X, \pi)$ we have $\widetilde{\pi}\left(t_{n}, x_{n}\right) \in V$ and $\widetilde{\pi}\left(t_{n}, x_{n}\right)=x^{k_{n}+}$ for some $k_{n}$. Thus $\tilde{\pi}\left(t_{n}, x_{n}\right)=I\left(q_{n}\right)$ for some $q_{n} \in \overline{B(E, \varepsilon)}$. Moreover, for 
each such $n$ there exists a $v_{n}<t_{n}$ such that there is no impulse point in the segment of trajectory $\widetilde{\pi}\left(\left(v_{n}, t_{n}\right), x_{n}\right)$.

For each $n$ take an $s_{n} \in\left(v_{n}, t_{n}\right)$ such that $t_{n}-s_{n} \rightarrow 0$. Define $p_{n}=$ $\widetilde{\pi}\left(s_{n}, x_{n}\right) \in \overline{B(E, \varepsilon)}$. We may assume (taking a subsequence if necessary) that $p_{n} \rightarrow p \in \overline{B(E, \varepsilon)}$. On the other hand, $q_{n}=\pi\left(t_{n}-s_{n}, p_{n}\right) \rightarrow \pi(0, p)=p$. Thus $p \in M$, since $q_{n} \in M$ for every $n$ and $M$ is closed.

However, $p=\lim _{n \rightarrow \infty} \widetilde{\pi}\left(s_{n}, x_{n}\right)$ and $x_{n} \rightarrow x$, so $p \in \widetilde{D}^{+}(x) \subset \widetilde{D}^{+}(A)$. According to Theorem 4.1, $p \in A$ and $p \in E$ as $\overline{B(E, \varepsilon)} \cap(A \backslash E)=\emptyset$. But $E$ is $I$-invariant, so $I(p) \in E$; on the other hand, $I$ is continuous, hence $I\left(q_{n}\right) \rightarrow I(p)$ and $I\left(q_{n}\right) \in V$, so $I(p) \in \bar{V}$ and $I(p) \notin E$. The contradiction finishes the proof.

The assumption that $E$ is an isolated component is essential, as can be seen in the following

5.5. Example. Consider the space $X=\left\{(x, y): 1 \leq x^{2}+y^{2} \leq 2\right\}$ and the dynamical system given by the equations $x^{\prime}=-y, y^{\prime}=x$. Define $A_{\alpha}=\left\{(x, y): x^{2}+y^{2}=\alpha^{2}\right\}$. Take $A=A_{1} \cup \bigcup\left\{A_{1+1 / n}: n=1,2, \ldots\right\}$. Note that $A_{1}$ is not isolated from $A \backslash A_{1}$. We take $M=\{(0,1)\} \cup\{0\} \times$ $\{1+1 / n: n=1,2, \ldots\}$. We now put $I((0,1))=(0,-1), I((0,2))=(0,-2)$ and $I((0,1+1 / n))=(0,-1-1 /(n-1))$. Then $A$ is stable, its component $A_{1}$ is $I$-invariant but $A_{1}$ is not stable. It is also easy to modify this example to have (STC) fulfilled; we take $M=\{0\} \times[1,2]$.

In Theorem 5.4 the assumption of $I$-invariance of $E$ cannot be replaced neither by the weaker assumption of $\widetilde{\pi}$-invariance nor by the condition that $E$ is a $\widetilde{\pi}$-attractor. We have the following

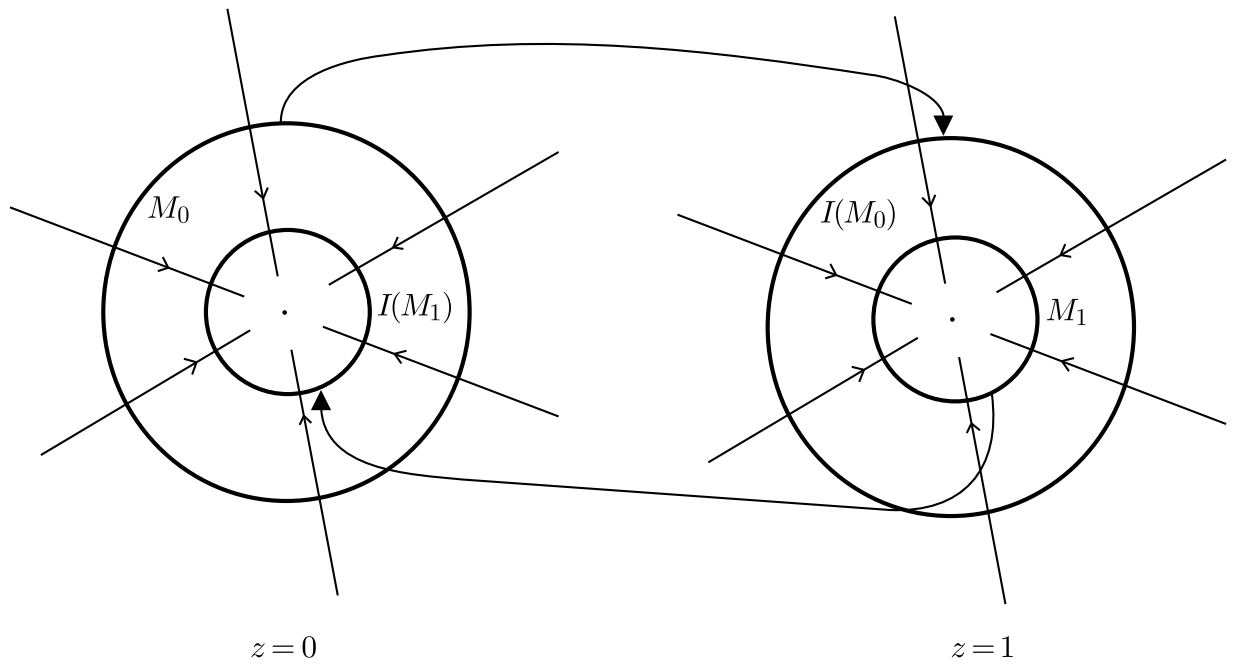

Fig. 3 
5.6. Example. Consider the space $X=\mathbb{R}^{2} \times\{0,1\}$ and the dynamical system given by the equations $x^{\prime}=-x, y^{\prime}=-y$ on $\mathbb{R}^{2} \times\{0\}$ and $\mathbb{R}^{2} \times\{1\}$, independently. Now let $M_{0}=\left\{(x, y, z): x^{2}+y^{2}=1, z=0\right\}, M_{1}=\{(x, y, z)$ : $\left.x^{2}+y^{2}=1 / 4, z=1\right\}, M=M_{0} \cup M_{1}$. We define $I(x, y, 0)=(x, y, 1)$ for $(x, y, 0) \in M_{0}$ and $I(x, y, 1)=(x, y, 0)$ for $(x, y, z) \in M_{1}$ (see Figure 3$)$. Take $A_{0}=\left\{(x, y): x^{2}+y^{2} \leq 1\right\} \times\{0\}, A_{1}=\left\{(x, y): x^{2}+y^{2} \leq 1\right\} \times\{1\}$, $A=A_{0} \cup A_{1}$. The set $A$ is $\tilde{\pi}$-stable, the component $A_{0}$ is a $\tilde{\pi}$-attractor and is positively $\tilde{\pi}$-invariant but it is not $\tilde{\pi}$-stable.

From Theorem 5.7 we have an immediate

5.8. Corollary. Assume that $X$ is locally compact, a compact set $A$ is $\widetilde{\pi}$-stable and $A$ has a finite number of components. Then any component $E$ of $A$ is $\tilde{\pi}$-stable provided it is I-invariant.

\section{References}

[BH] N. P. Bhatia and O. Hajek, Local Semi-Dynamical Systems, Lecture Notes in Math. 90, Springer, 1970.

[BS] N. P. Bhatia and G. P. Szegő, Stability Theory of Dynamical Systems, Springer, 2002.

[C1] K. Ciesielski, Sections in semidynamical systems, Bull. Polish Acad. Sci. Math. 40 (1992), 297-307.

[C2] -, On semicontinuity in impulsive dynamical systems, this volume, 71-80.

[K1] S. Kaul, On impulsive semidynamical systems, J. Math. Anal. Appl. 150 (1990), $120-128$.

[K2] - On impulsive semidynamical systems II, Recursive properties, Nonlinear Anal. 16 (1991), 635-645.

[LBS] V. Lakshmikantham, D. D. Bainov and P. S. Simeonov, Theory of Impulsive Differential Equations, World Sci., 1989.

[NS] V. V. Nemytskiu and V. V. Stepanov, Qualitative Theory of Differential Equations, Princeton Univ. Press, 1960.

[P] A. Pelczar, Introduction to the Theory of Differential Equations, Part II, Bibl. Mat. 67, Polish Sci. Publ., 1989 (in Polish).

[V] J. de Vries, Elements of Topological Dynamics, Math. Appl. 257, Kluwer, 1993.

Krzysztof Ciesielski

Mathematics Institute

Jagiellonian University

Reymonta 4

30-059 Kraków, Poland

E-mail: ciesiels@im.uj.edu.pl 